\title{
Les orientations du Livre blanc municipal : Incidences envisagées sur la gestion des petites municipalités
}

\author{
Caroline Lépine $^{\mathrm{a}}$ et Étienne Charbonneau ${ }^{\mathrm{b}}$
}

\section{INTRODUCTION}

Les directeurs généraux des municipalités québécoises seront des acteurs clés dans l'exécution de ces engagements, plus particulièrement dans les plus petites municipalités où le personnel cumule les fonctions.

À la suite du Sommet sur le milieu municipal de mars 2011, l’Union des municipalités du Québec a lancé un grand chantier de réflexion collective afin de proposer au gouvernement provincial des solutions aux principaux enjeux municipaux. Au terme de cet exercice, plusieurs engagements ont été identifiés et ces derniers auront des incidences directes sur la gestion du territoire. Les directeurs généraux des municipalités québécoises seront des acteurs clés dans l'exécution de ces engagements, plus particulièrement dans les plus petites municipalités où le personnel cumule les fonctions. L'article vise à envisager quelques-unes des conséquences des conclusions du Livre blanc municipal sur le travail quotidien des directeurs

\section{PRÉSENTATION DE L'ENQUÊTE}

Les données présentées sont issues d'une enquête complétée en 2012 sur le travail de gestion des directeurs généraux et secrétaires-trésoriers. Cette enquête a été réalisée par Yvon Douville, en collaboration avec l'Association des directeurs généraux des municipalités du Québec, comme projet d'intervention au programme de maîtrise à l'École nationale d'administration publique. Le questionnaire comportait neuf questions visant à décrire d'une part le travail de gestion, notamment les secteurs d'activité, les actions, les outils et les intervenants. D'autre part, il avait pour but de dresser un portrait non seulement des répondants, mais aussi des municipalités de moins de 5000 habitants. Les municipalités sont issues de l'ensemble des régions administratives du Québec, à l'exception des régions de Laval et Montréal, ne regroupant aucune généraux des municipalités de moins de 5000 habitants, alors qu'elles représentent plus de $80 \%$ du nombre total de municipalités québécoises.

Pour ce faire, il est proposé de mettre en relation les résultats d'une enquête visant les réalités de la gestion des petites municipalités au contenu du Livre blanc municipal adopté la même année. L'enquête, sous la forme d'un sondage, a été réalisée auprès des directeurs généraux et secrétaires-trésoriers membres de l'Association des directeurs généraux des municipalités du Québec. Les répondants au questionnaire sont issus de 367 municipalités locales québécoises dont la population est inférieure à 5000 habitants.

Les réalités liées au travail de gestion municipale seront identifiées au moyen de quatre composantes d'une enquête. Par la suite, le Livre blanc municipal et ses incidences sur la gestion municipale seront présentés. Finalement, un aperçu des incidences envisagées des engagements du Livre blanc municipal sur les réalités de gestion est proposé.

municipalité détenant les caractéristiques souhaitées. Les régions les plus présentes sont ChaudièreAppalaches (58 municipalités), le Bas-SaintLaurent (48) et l'Estrie (32); tandis que les régions du Saguenay-Lac-Saint-Jean (12), de Lanaudière (15) et de la Mauricie (17) sont moins représentées.

$\mathrm{Au}$ niveau de la population, notons que le quart (25\%) des 367 municipalités visées détiennent 500 habitants et moins; et plus de $80 \%$ des municipalités possèdent 2000 habitants et moins. Quant au budget, $81 \%$ des municipalités possèdent un budget de 3 millions de dollars ou moins; un nombre considérable de municipalités (27\%) doivent réaliser leurs activités avec un budget maximal d'un million. Ces municipalités se caractérisent aussi par le faible nombre d'employés 
permanents. Plus de la moitié d'entre elles dénombrent seulement un à cinq employés permanents. Lorsque l'on inclut les municipalités ayant 10 employés permanents et moins, cette proportion augmente à $81 \%$.

\section{UN PORTRAIT DES RÉALITÉS DE GESTION DES DIRECTEURS GÉNÉRAUX}

\section{Les multiples réalités des municipalités ont pour effet de diversifier les fonctions des directeurs généraux d'une municipalité à l'autre.}

Les multiples réalités des municipalités ont pour effet de diversifier les fonctions des directeurs généraux d'une municipalité à l'autre. Par exemple, nombre d'entre eux (217) combinent d'autres fonctions telles que celle de secrétaire (greffe) et/ou de trésorier. Lorsqu'interpellés sur la division de leur fonction, $25 \%$ des répondants évaluent que la gestion, à proprement dit, correspond à plus de la moitié de leur emploi du temps. De plus, seulement quatre d'entre eux estiment consacrer leurs activités entièrement à la gestion. Les tâches liées au secrétariat et à la trésorerie représentent chacune $25 \%$ des activités pour plus de la moitié des répondants.

Plusieurs composantes de la gestion des directeurs généraux ont été abordées par l'enquête. Toutefois, nous nous concentrerons sur quatre d'entre elles, soient les secteurs d'activité, les actions quotidiennes, les interactions et les outils de gestion. Le graphique 1 regroupe les résultats de trois composantes. La composante « secteurs d'activité » vise à établir le niveau d'implication des répondants dans 14 secteurs d'activités, la composante " actions quotidiennes » cherche à établir les actions composant la fonction de gestion à travers 9 verbes d'action, tandis que la composante " outils de gestion » vise à déterminer le niveau d'utilisation de cinq outils. Ces trois composantes ont été évaluées sur une échelle allant de 1 à 4 (pas du tout, un peu, passablement, beaucoup). La quatrième composante, dont les résultats sont illustrés au graphique 2, concerne les interactions avec les différents acteurs. Vingt-et-un acteurs sont identifiés. Ils peuvent être divisés en cinq grandes catégories d'intervenants, c'est-à-dire (1) les intervenants politiques, (2) les intervenants municipaux, (3) les intervenants gouvernementaux, (4) les intervenants professionnels et (5) les intervenants populaires. Cette composante a été évaluée sur une échelle allant de 1 à 5 (ne connais pas, liens peu fréquents et peu importants, liens peu fréquents mais importants, liens fréquents peu importants, liens fréquents et importants).

Figure 1 Évaluation de l'intensité des composantes de gestion des directeurs généraux liées aux secteurs d'activité, aux actions quotidiennes et aux outils de gestion

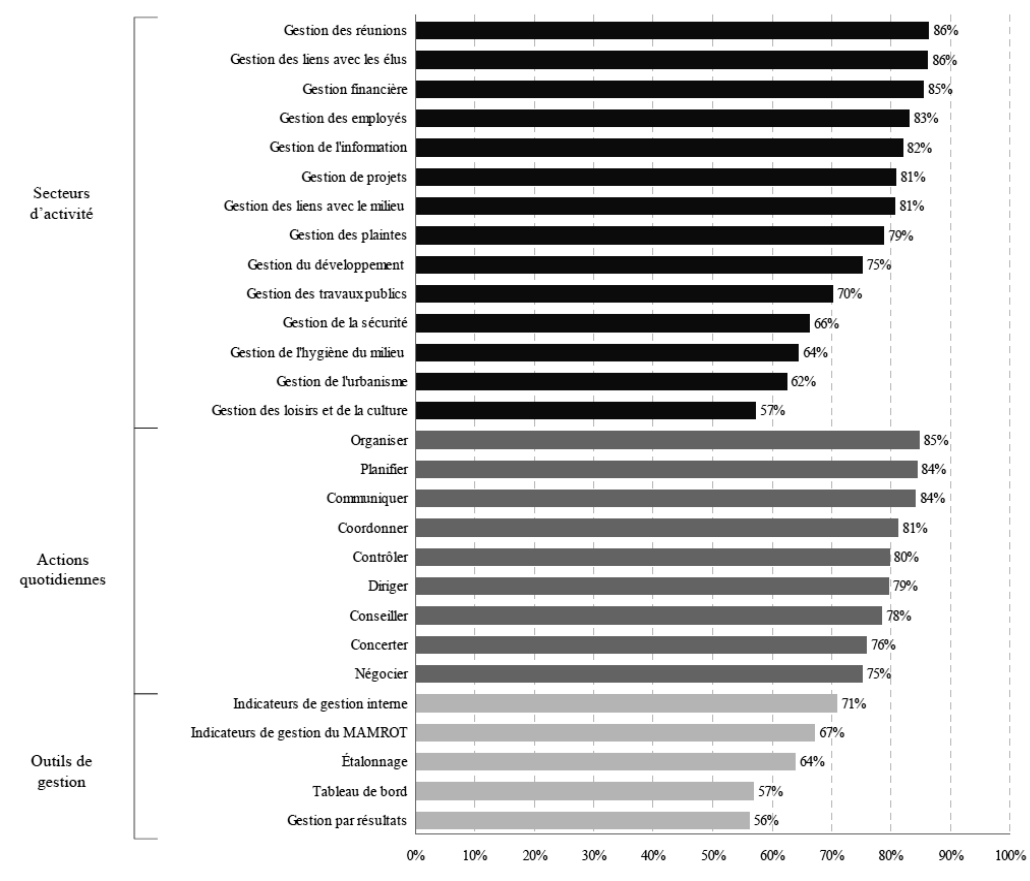


L'enquête a révélé que la gestion des réunions, des liens avec les élus et des finances sont les secteurs d'activités où les directeurs généraux sont les plus impliqués. La gestion de la sécurité, de l'hygiène du milieu, de l'urbanisme et des loisirs et de la culture constituent les secteurs où l'implication des directeurs généraux n'est pas aussi importante sans pour autant y être totalement absente. Le phénomène peut se justifier, par exemple, par la présence de professionnels dans ces secteurs d'activités.

Le travail de gestion peut aussi être décomposé en plusieurs types d'actions. Parmi les actions proposées, les répondants ont identifié l'organisation, la planification et la communication comme leurs principales actions au quotidien. À l'opposé, les actions liées à la concertation et à la négociation apparaissent être les plus secondaires.

Divers outils peuvent être mis à profit dans le cadre du travail de gestion des directeurs généraux. Le recours à des indicateurs de gestion développés par chacune des municipalités est l'outil le plus fréquemment utilisé suivi par les indicateurs de gestion du MAMROT. Le tableau de bord et la gestion par résultats sont quant à eux les outils les moins exploités.

La gestion municipale implique inévitablement des interactions avec différents acteurs. De façon générale, les liens entre les directeurs généraux et les intervenants politiques sont les plus fréquents et considérés importants, plus particulièrement lorsqu'il s'agit des liens avec le maire de la municipalité. Par ailleurs, les interactions avec les employés municipaux apparaissent être tout aussi fréquentes et importantes. Bien que les liens avec les intervenants gouvernementaux et les intervenants professionnels soient moins fréquents, leur importance n'est pas sous-estimée par les directeurs généraux. Certains résultats attirent plus particulièrement notre attention, notamment le peu d'importance accordée aux interactions avec les journalistes et les intervenants populaires tels que les comités. À noter que lorsqu'il s'agit d'un citoyen individuel, la fréquence et l'importance des interactions sont fortement plus élevées.

Figure 2 - Évaluation de l'intensité des interactions des directeurs généraux

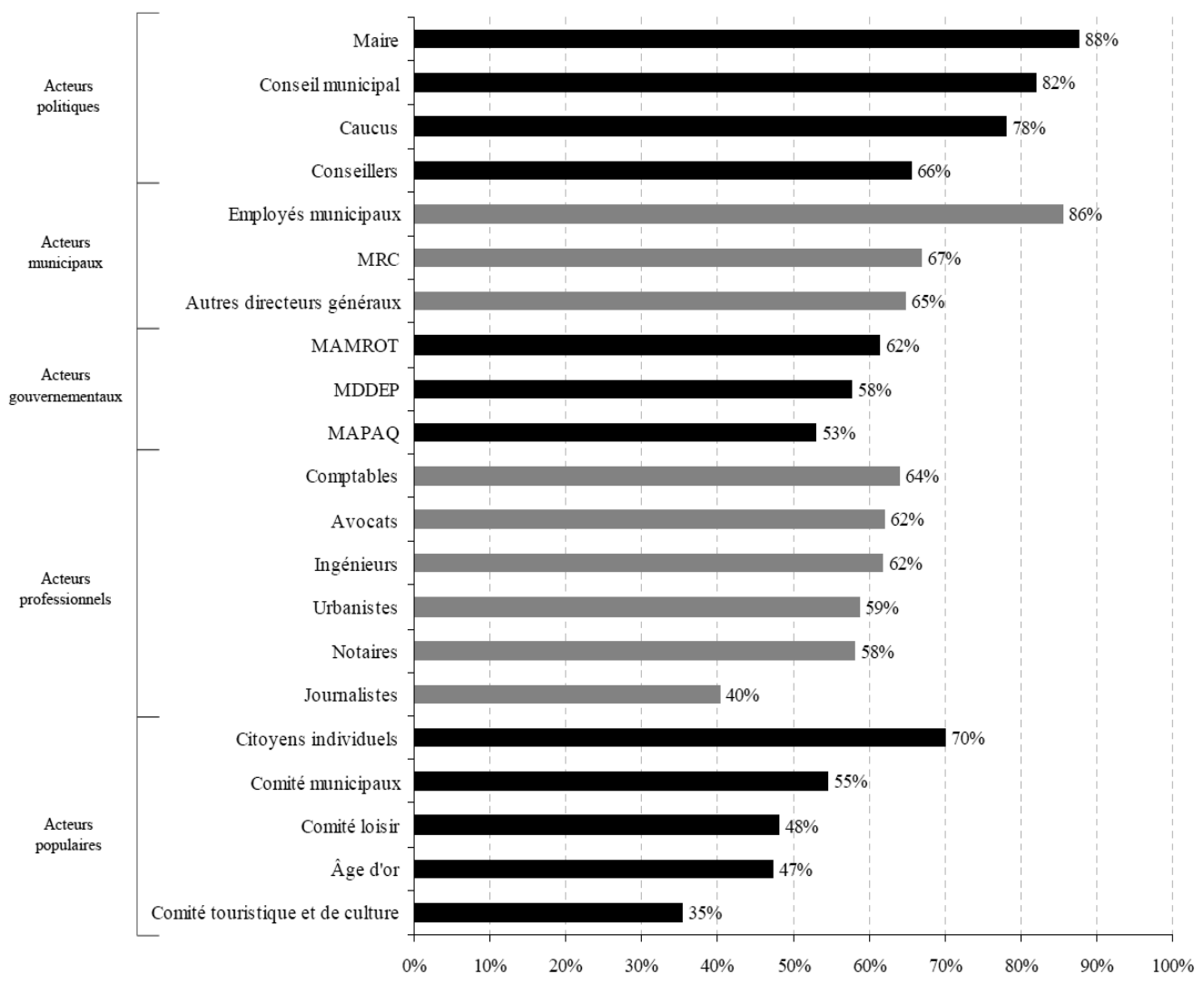

Organisations \& territoires (2015) 
$\mathrm{Au}$ final, le sondage a permis de dégager deux grandes réalités du travail de gestion des directeurs généraux dans les petites municipalités. Dans un premier temps, la gestion des municipalités se caractérise au quotidien par la gestion des relations fréquentes avec les élus, les employés municipaux et les citoyens individuels exigeant des habiletés de communication et une gestion efficiente des réunions. Deuxièmement, la gestion de ces munici- palités comporte des activités d'organisation et de planification en lien notamment avec la gestion financière impliquant des relations non seulement avec les élus, mais aussi avec des représentants gouvernementaux, tels que le MAMROT. Ceci est particulièrement dû au contexte structurel de la gestion du territoire et à la réforme entreprise par le gouvernement provincial en 2004.

\section{LE LIVRE BLANC MUNICIPAL ET SES INCIDENCES SUR LA GESTION MUNICIPALE ${ }^{1}$}

Le projet de Livre blanc municipal a été lancé en novembre 2011 afin de développer une vision renouvelée des municipalités. Au-delà de ce premier objectif, le Livre blanc vise aussi à renforcer la confiance du public envers les municipalités et ses acteurs. Issu notamment de la demande formulée au gouvernement du Québec de reconnaître le statut des municipalités comme gouvernement local, le projet a été développé selon une démarche se voulant inclusive et collective caractérisée par une vaste consultation des nombreuses parties prenantes. Au cours de l'exercice, deux enjeux ont été identifiés : (1) les limites des compétences municipales, et (2) la précarisation des finances municipales. Au final, deux orientations ont été élaborées;

\section{Orientation 1-Rapprocher la décision du citoyen}

Cette première orientation propose l'adoption d'une Charte des municipalités visant à moderniser le cadre législatif afin de munir les municipalités d'une autonomie locale dans l'offre d'une réponse adaptée aux besoins évolutifs de leur population. La Charte a pour but de mettre de l'avant divers principes tels que la subsidiarité, la démocratie, la souplesse et l'efficience.

Orientation 2 -Adapter la fiscalité municipale aux responsabilités du XXI siècle

La deuxième orientation tente de répondre à de nombreuses réalités touchant la fiscalité des municipalités. Un meilleur contrôle des dépenses publiques constitue une première solution afin d'agir sur la rémunération, la gestion contractuelle, les régimes de retraite et l'endettement. La nécessité de faire des choix soutenant le développement durable, c'est-à-dire des choix favorisant l'écologie et la cohésion sociale, se présente comme un moyen de créer de la richesse.
Les incidences de la mise en œuvre des solutions identifiées au Livre blanc sont nombreuses. D'ailleurs, il s'avère probablement impossible, à l'heure actuelle, de toutes les identifier. Néanmoins, trois ont capté notre attention.

\section{Incidence 1 -La participation citoyenne}

Comme il est souligné au Livre blanc municipal, la participation citoyenne se fonde tout d'abord sur la volonté des citoyens. En ce sens, aucune municipalité ou gouvernement ne peut l'imposer. Malgré tout, les municipalités devront se charger de l'organiser et de l'encourager. Pour ce faire, elles devront se munir d'outils et de mécanismes nouveaux et/ou réviser certaines de leurs pratiques actuelles, souvent bien ancrées dans la culture organisationnelle. Par exemple, afin d'assurer le succès des démarches de participation citoyenne, les municipalités devront s'assurer de transmettre l'information de manière à ce qu'elle puisse être compréhensible pour le citoyen. Il s'agit ici d'un enjeu majeur lié au domaine des communications des municipalités.

\section{Incidence 2 -La gestion de contrats}

Les contrats de fourniture de services et de ressources matérielles requièrent une large part des budgets des municipalités. En lien avec les récents événements, les pratiques générales de gestion contractuelle ont eu pour effet d'altérer le lien de confiance entre la population et les municipalités. Il est donc inévitable que des mesures soient envisagées afin de régulariser cette situation. Parmi ces solutions, le Livre blanc municipal propose de miser sur la transparence grâce à la mise en place entre autres d'un bureau d'évaluation des prix. Ce dernier aurait de nombreux rôles; notamment un rôle de vérification et un rôle-conseil. Les incidences 
pour les municipalités portent ainsi sur la nécessité de réviser les processus liés aux appels d'offres et à l'octroi des contrats afin d'y inclure cette nouvelle instance.

\section{Incidence 3-La transparence et le rendement}

La transparence et le rendement sont visés par les deux orientations du Livre blanc municipal; c'està-dire qu'ils doivent être pris en compte tant au niveau de la prise de décision que dans la gestion des fonds publics. Mais au-delà des principes généraux visant l'accessibilité à l'information, la gestion municipale devra aussi revoir ses processus de reddition de comptes, car elle devra répondre plus spécifiquement à la population. En ce sens, l'information devra être présentée de manière à ce que le citoyen puisse comprendre et suivre les décisions et les actions des administrateurs municipaux.

\section{LES INCIDENCES ENVISAGÉES DU LIVRE BLANC MUNICIPAL SUR LE TRAVAIL DE GESTION DES DIRECTEURS GÉNÉRAUX}

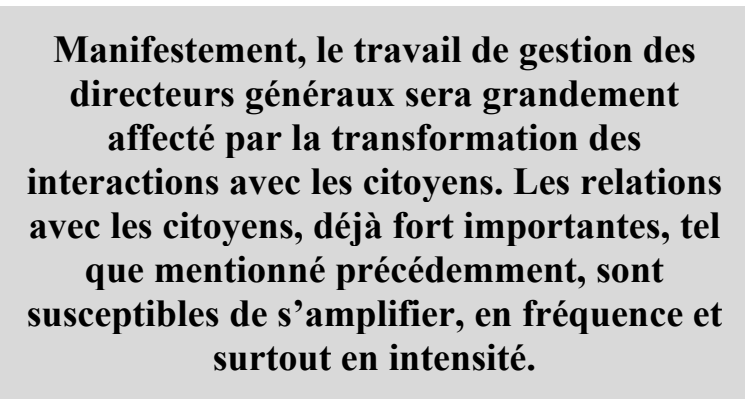

Il a été souligné précédemment que les fonctions des directeurs généraux des petites municipalités sont la gestion, le secrétariat et la trésorerie. La mise en œuvre des engagements du Livre blanc municipal aura des incidences sur chacune d'entre elles. Par exemple, les modifications au niveau des processus de la gestion contractuelle influenceront les activités de gestion et les activités de trésorerie. De plus, pour les activités de secrétariat, les objectifs de transparence feront que les informations sur la gestion et les décisions devront être largement disponibles en ligne.

Manifestement, le travail de gestion des directeurs généraux sera grandement affecté par la transformation des interactions avec les citoyens. Les relations avec les citoyens, déjà fort importantes, tel que mentionné précédemment, sont susceptibles de s'amplifier, en fréquence et surtout en intensité. Dans leur vie quotidienne, les citoyens prennent l'habitude à travers les médias sociaux, d'échanger et d'interagir. Il ne suffira plus à la municipalité de bien servir et de bien communiquer.

Ainsi, au-delà des principes de gestion de l'information, la participation citoyenne implique l'instau- ration d'un dialogue entre la municipalité et sa population. Il ne s'agit plus de répondre aux exigences de la Loi sur l'aménagement et l'urbanisme visant la transmission de l'information et la tenue de référendum. De nouveaux modèles de participation, tels que celui de la ville de Québec par l'entremise de ses conseils de quartier soutenant les principes de gouvernance ou celui de Montréal avec la création de l'Office de consultation publique de Montréal dont la mission vise à tenir des débats publics $^{2}$, pourront être mis en place. De ce fait, les directeurs généraux devront développer leurs habiletés en communication et en concertation. L'enjeu principal de la participation citoyenne réside ainsi dans la distinction entre la transparence et la participation. Alors que la première vise principalement la transmission de l'information, la deuxième réfère à la contribution de la population dans le processus décisionnel ${ }^{3}$.

L'impartition n'est pas largement pratiquée dans les municipalités visées par l'enquête, considérant que plus des trois quarts d'entre elles opèrent leur service incendie, réseau d'eau potable et réseau des eaux usées en régie interne. Cependant, la mise sur pied du bureau d'évaluation des prix imposera tout de même des changements dans le processus d'octroi des contrats. De ce fait, fort est à parier que les directeurs généraux devront s'impliquer encore davantage dans certains secteurs d'activité tels que la gestion financière, la gestion de projet et la gestion des liens avec les divers intervenants. À cet effet, comme le bureau d'évaluation des prix deviendra un nouvel interlocuteur, les directeurs généraux seront responsables d'établir les liens entre leur municipalité et cette nouvelle instance. 
Le rôle de vérification que semble accorder le Livre blanc municipal au bureau d'évaluation des prix laisse présager l'imposition de règles et de processus additionnels.

Le rôle de vérification que semble accorder le Livre blanc municipal au bureau d'évaluation des prix laisse présager l'imposition de règles et de processus additionnels. De plus, toujours en lien avec la création du bureau d'évaluation des prix, de nouveaux indicateurs de gestion seront probablement imposés pour le suivi des activités contractuelles des municipalités. Les enjeux principaux de la gestion contractuelle résident donc non seulement dans un éventuel alourdissement de la procédure et des délais de réalisation des travaux, mais aussi dans la réticence des directeurs généraux d'utiliser ces indicateurs dans leur gestion quotidienne. Autrement dit, les informations seront recueillies uniquement dans le but de satisfaire l'instance supérieure et non comme outil interne afin d'assurer une gestion adéquate des finances municipales. Ce phénomène a notamment été perçu à la suite de la réforme entreprise par le gouvernement provincial en 2004 imposant des mesures de performance à toutes les municipalités de la province. ${ }^{4}$

La communication et le contrôle seront les deux actions les plus touchées par les objectifs liés à la transparence et au rendement. D'une part, la gestion de l'information devra être révisée afin d'assurer que l'information est non seulement disponible, mais aussi qu'elle soit compréhensible pour les

\section{CONCLUSION}

Pour les petites municipalités, le succès de la mise en œuvre des orientations du Livre blanc municipal dépendra grandement de l'apport des directeurs généraux dans la gestion des activités quotidiennes au sein de leur organisation, au moment où plusieurs songent à prendre leur retraite.

Pour les petites municipalités, le succès de la mise en œuvre des orientations du Livre blanc municipal dépendra grandement de l'apport des directeurs généraux dans la gestion des activités quotidiennes citoyens. D'autre part, des indicateurs de gestion devront être utilisés afin de présenter la performance de l'administration municipale, de préférence modulés par l'intensité des bénéfices reçus, selon la localisation ${ }^{5}$. À cette fin, plusieurs outils de gestion peuvent leur être utiles.

Il faudra aussi prévoir une transformation dans le type de communication. Aujourd'hui, les directeurs généraux interagissent principalement avec des professionnels et des élus politiques; demain, ils devront interagir de façon plus soutenue avec la population, non seulement avec les citoyens individuellement, mais aussi divers groupes. Il s'agit donc de développer leurs habiletés à vulgariser des informations techniques, à concerter et à rassembler. À cette fin, les nouvelles technologies se présentent comme des outils indispensables. Alors que le modèle de « ville intelligente " gagne en popularité, les technologies informatiques offrent des possibilités de prestation des services aux citoyens, mais aussi dans le partage de l'information et le dialogue. À noter que ces technologies sont aussi à la disposition des petites municipalités ${ }^{6}$. Pour ce faire, les directeurs généraux doivent tenir à jour leurs connaissances technologiques et suivre l'évolution des technologies disponibles et propices à la gestion du territoire.

Les directeurs généraux doivent tenir à jour leurs connaissances technologiques et suivre l'évolution des technologies disponibles et propices à la gestion du territoire. au sein de leur organisation, au moment où plusieurs songent à prendre leur retraite.

D'ailleurs selon le portrait des membres de l'ADMQ pour 2012, $13 \%$ des membres déclarent prévoir quitter leur fonction dans un horizon de cinq ans. Cette proportion doublant (27\%) lorsque l'on examine les départs prévus au cours des dix prochaines années. Il est donc évident que la profession se renouvellera dans les prochaines années laissant place à une nouvelle génération de directeurs généraux. À cet effet, ajoutons que quinze municipalités sont dirigées par des directeurs de moins de 34 ans. 
En définitive, il semble inévitable que les réalités des directeurs généraux des plus petites municipalités seront affectées par les pistes de solutions proposées par le Livre blanc municipal. Dans le contexte de la mise en cuvre du Livre blanc municipal, nous avons identifié quatre habiletés qui devront être mises à profit par les directeurs généraux : la communication, la concertation, le rôleconseil et le contrôle. La communication visant la réception, le traitement et la diffusion de l'information sera capitale en lien avec les objectifs de transparence et de participation citoyenne. La participation citoyenne sera aussi grandement influencée par les habiletés de concertation des directeurs généraux. Au niveau du rendement, les habiletés visant le contrôle devront être mises de l'avant afin d'assurer un suivi des activités. Finalement, les directeurs généraux seront appelés à conseiller leurs élus et leurs employés en matière de gestion contractuelle.

\section{La communication visant la réception, le traitement et la diffusion de l'information sera capitale en lien avec les objectifs de transparence et de participation citoyenne.}

\section{BIBLIOGRAPHIE}

${ }^{1}$ Union des municipalités du Québec (2012). Livre blanc municipal - L'avenir à un lieu, Montréal, Québec, 78 pages. [en ligne http://www.livreblancmunicipal.ca] ${ }^{2}$ Bherer, L. (2011). « Les trois modèles municipaux de participation publique au Québec », Télescope, vol. 17, $\mathrm{n}^{\circ} 1, \mathrm{p} .157-171$.

${ }^{3}$ Welch, E.W. (2012). "The Relationship between Transparent and Participative Government: A Study of Local Governments in the United States", International Review of Administrative Sciences, vol. 78, $\mathrm{n}^{\circ}$ 1, p.93-115.

${ }^{4}$ Charbonneau, É. et Nayer, G. (2012). "Barriers to the Use of Benchmarking Information: Narratives from Local Government Managers", Journal of Public Management \& Social Policy, vol. 18, n², p.25-47.

${ }^{5}$ Divay, G. (2012). «L'engagement civique local : de l'incantation générique à la valorisation segmentée » Administration publique du Canada, vol. 55, $\mathrm{n}^{\circ} 4, \mathrm{p} .505-530$.

${ }^{6}$ Charbonneau, É. et Divay, G. (2014). « La municipalité, le ferment d'une "ville intelligente"? ", Le Sablier, vol. $21, \mathrm{n}^{\circ} 1, \mathrm{p} .18-19$.

\section{NOTES}

${ }^{a}$ Caroline Lépine est conseillère en aménagement à la Ville de Montréal et étudiante au doctorant à l'École nationale d'administration publique. Elle s'intéresse particulièrement à l'évaluation des politiques urbaines et à la participation citoyenne à l'échelle locale.

'Étienne Charbonneau, Ph.D., est professeur adjoint en management public à l'École nationale d'administration publique. Il est membre du Centre de recherche sur la gouvernance (CERGO). Ses recherches traitent de la mesure de la performance et de la satisfaction des citoyens. 


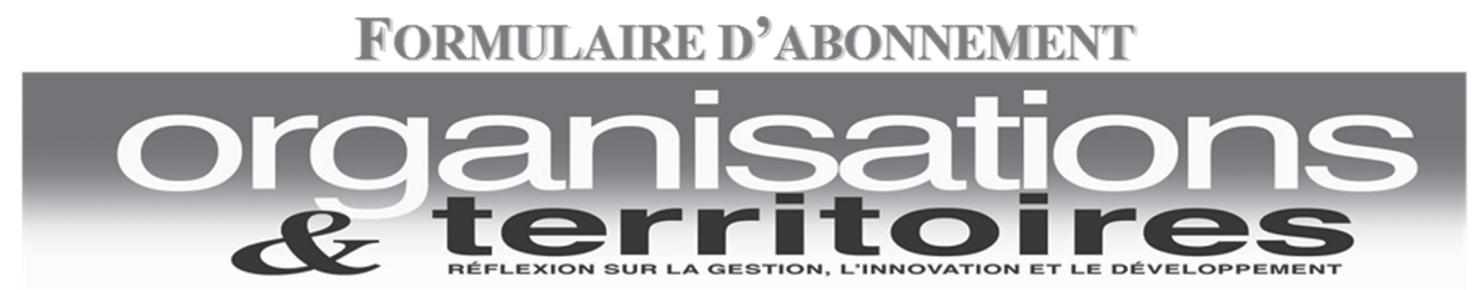

Je désire m'abonner à la revue Organisations et territoires et je joins un chèque au montant de : $\$$

Nouvel abonné $(\sqrt{ })$ :

Renouvellement $(\sqrt{ })$ :

Date :

Abonnement institutionnel (6 numéros) 103,48 \$ (Toutes taxes incluses)

Abonnement individuel (6 numéros) 66,69 $\$$ (Toutes taxes incluses)

Abonnement étudiant (6 numéros) 48,29\$ (Tx inc.) (Envoyer photocopie carte étud.)

Abonnement gouvernemental (6 numéros) 90,00\$ (Fournir certificat d'exemption des taxes)

Abonnement hors Canada $\quad$ (6 numéros) $145,00 \$ \quad(110 \$+35 \$$ frais de poste) (sans taxes)

Tous les abonnements sont d'une durée de deux ans

- J'aimerais que mon abonnement débute avec le DERNIER numéro paru, soit :

Volume $\mathrm{N}^{\circ}$

- J'aimerais que mon abonnement débute avec le PROCHAIN numéro à paraittre, soit :

Volume $\mathrm{N}^{\circ}$

Faites-nous parvenir vos coordonnées :

Nom :

Organisation :

Adresse :

Téléphone :

Télécopieur :

Courriel :

Adresse d'expédition : $\quad$ Revue Organisations et territoires Université du Québec à Chicoutimi 555, boulevard de l'Université, bureau H6-1430

Chicoutimi (Québec) CANADA G7H 2B1

Téléphone : (418) 545-5011, poste 4530 Télécopieur : (418) 693-9072

Courriel : revueot@uqac.ca Site Web : www.uqac.ca/revueot 